\title{
Effective leadership strategies improve length of stay and patient outcomes in total arthroplasty surgery
}

\author{
S Radha ${ }^{1,2}$, I Afzal ${ }^{3 *}$ and W Radford ${ }^{1}$ \\ ${ }^{1}$ Chelsea and Westminster Hospital, London, United Kingdom \\ ${ }^{2}$ Greenwich School of Management, London, United Kingdom \\ ${ }^{3}$ South West London Elective Orthopaedic Centre, London, United Kingdom
}

\begin{abstract}
Prolonged length of stay (LOS) leads to increased costs and reduced income for the hospital, as well as increased costs to the patient. This, in turn, leads to provision of poor quality of care by healthcare organization due to lack of resources. This research aims to review the causes of longer stay of patients in the hospital post Total Hip Arthroplasty (THA) or TJA (Total Joint Arthroplasty) and the effectiveness of the leadership in reducing the length of stay of patients in the hospital.

Mixed Method technique has been adopted to analyse the data. Primary data for the qualitative technique has been obtained using Interviews of the experts in the discharge process of the hospital. Quantitative data was collected from the hospital records for two periods: first for the period $6^{\text {th }}$ October 2016 to $27^{\text {th }}$ March 2017 , which is prior to interventions and second period from $27^{\text {th }}$ March 2017 to $6^{\text {th }}$ October 2017, which was the period post intervention.

Research findings show that effective leadership encourages effective communication between patients, their families and admitting team that leads to avoid discharge delays. Communication by the team leader with the patients and their families is the most effective strategy in quick discharge. The study shows that one channel of communication prior to surgery from the leader of the team will be most effective strategy. Further, effectiveness of the leader is essential to facilitate effective channels of communication regarding discharge process. Flexible leadership is most suited to the healthcare sector.
\end{abstract}

\section{Introduction}

A Total Hip Arthroplasty (THA) is an effective procedure to manage arthritis but also to restore quality of life, which typically involves recreational and physically demanding activities. Most of the cost of THA is involved in the Length of Stay (LOS) in hospital, which healthcare providers naturally seek to shorten. In the past decade, the length of in-patient stay following THA has declined from an average of three weeks to four days [1-3].

Mostly recently, emphasis has been placed on Enhanced Recovery After Surgery (ERAS) aiming to reduce the LOS potentially moving towards day case surgery, discharge within 24 hours, following THA. With the growing emphasis on cost efficiency in the health care system, a measure of success after THA for both patients and hospitals has also shifted towards rapid recovery and shorter LOS. Hospitals and healthcare organisations have therefore been encouraging research efforts on improvements in programmes such as ERAS [4].

In a fluid, complex and dynamic environment where patients discharge from hospital occurs, effective leadership has been seen to be pivotal in attaining successful outcome [5]. Effective strategies to identify and overcome barriers for a timely discharge can help in reducing patients LOS, which in turn reduces hospital cost and expenditure [6]. A recent systematic review has found a correlation between leadership, the quality of care and patients outcome. Hospital leadership is considered a core element within any health care organisation. Effective leadership in managing a discharge plan following the admission of patients to the hospital has been poorly studied within the literature. The role of a leader, in order to facilitate early patient discharge with minimal or no cost to healthcare organisation is imperative and therefore essential to be studied and explored.

This study aims at exploring the value of effective leadership in reducing the length of in-patient stays following a THA.

\section{Methods}

We undertook a mixed method, quantitaive and qualitative, research study. Data for the quantitative analysis was collected from hospital records for a period of one year whereas data for qualitative analysis is collected through interviews of the selected participants from three hospitals in UK.

\section{Qualitative research}

In selecting participants, the researcher ensured the participants had direct experience with patient discharge planning; they had spent a minimum of two years leadership position of employment within the NHS and had taken up roles for strategic implementation to reduce the LOS following THA.

After ensuring the participants met the research inclusion criteria and ensuring their interest in participation, informed consent was gained from participants who wished to take part in the study.

${ }^{\star}$ Correspondence to: Irrum Afzal, South West London Elective Orthopaedic Centre, London, United Kingdom, E-mail: Irrumaafzal@gmail.com

Received: December 09, 2019; Accepted: December 26, 2019; Published: January 03, 2020 
The participants were informed that they have right not to participate in the study. They could withdraw the participation at any point of time and had the right to refuse to answer any of the interview questions.

If the participants wanted to withdraw from the study at any time they were able to do so without having to give a valid reason. The participants need to inform the researcher at any point of time, if they wanted to terminate their participation. Data collected prior to withdrawal will still be used. (Table 1):

Each participants was asked the below question as part the study

\section{Quantitative research}

114 patients were recruited into this study. The data was collected for the period; October 2016 to October 2017 from the records of the hospital under the study. Data collected contained details of the length of stay of the patients, age, gender, ASA grade, readmissions and reasons for the delays in discharge. Data was collected for two periods: first for the period $6^{\text {th }}$ October 2016 to $27^{\text {th }}$ March 2017, which is prior to interventions and second period from $27^{\text {th }}$ March 2017 to $6^{\text {th }}$ October 2017, which was the period post intervention. Statistical analysis of the data collected was carried out to get the analysis done.

No formal ethical approval was necessary for the quantitative part of the study. The data was readily available and did not include any intervention to patients or participants. The National Code on Clinical Trials has reported that ethics approval is not necessary for real retrospective studies (National Code on Clinical Researches, 2011).

\section{Results}

Results from this study showed, three different responses to the question. Ten respondents were grouped to Respondent Group 1 (R1), ten respondents were grouped to Respondent Group (R2) and ten to Respondent Group 3 (R3) for "What type of leadership strategies will be most effective in making the discharge process efficient?". R1 reported that, despite the availability of the discharge process, effective communication between patients, their families and the admitting team plays an important role in avoiding discharge delays. R2 reported that discharge document pathways give information on overall patients journey but that does not cover additional questions patients and their family may have. Therefore, this document should not replace the need for direct communications between patients and hospital staff. R3 reported that information leaflets and booklets sometimes may not be read or understood by patients and their families. Therefore, they

Table 1. A list of questions asked as a part of the qualitative aspect of the study

\begin{tabular}{|c|l|l|}
\hline No. & \multicolumn{1}{|c|}{ Question } & $\begin{array}{c}\text { Response of the } \\
\text { participants }\end{array}$ \\
\hline 1 & $\begin{array}{l}\text { What is the main leadership strategy for making the discharge } \\
\text { process timely? }\end{array}$ & \\
\hline 2 & $\begin{array}{l}\text { Describe the discharge process } \\
\text { employed in the discharge of the patient under the present } \\
\text { leadership strategy. }\end{array}$ & \\
\hline 3 & $\begin{array}{l}\text { What leadership strategies would you use in a department to } \\
\text { reduce incidents of delayed discharges? }\end{array}$ \\
\hline 4 & $\begin{array}{l}\text { What type of leadership strategies will be most effective in } \\
\text { making the discharge process efficient? }\end{array}$ \\
\hline 5 & $\begin{array}{l}\text { What aspects of leadership would you change to better facilitate } \\
\text { the discharge process in your department? }\end{array}$ \\
\hline 6 & $\begin{array}{l}\text { What modifications would you like to introduce in the leadership } \\
\text { strategies being used in your department to reduce the delay in } \\
\text { discharge and improve the profitability of the hospital? }\end{array}$ \\
\hline
\end{tabular}

were all in agreement that, effective leadership is required to facilitate an appropriate channel of communication with patients and patients' families regarding the discharge process. Ganong \& Coleman (2014), stressed upon the need for clear and direct dialogue between the hospital team and the patients to facilitate an effective discharge process [7].

Involvement and communication with patients' family members were seen as an extremely important aspect of the discharge process and planning. R3 reported that significant discharge delay took place at their hospital in one particular period of time due to ineffective communication with the patient's family. In an specific issue of preparing transport from families, availability of facilities at home and availability of caregivers have been an element of discharge failure in several cases at the hospital of R3. A study performed by Greyden et al in 2012 showed poor patient and family communication was discovered as one of the prime issues leading to delayed discharge [8]. Effective leadership needs to employ string strategies to facilitate this communication process smoothly. It is therefore fair to suggest in order to avoid discharge delays, decisions by the leader, aimed at discharge instructions are conveyed and understood by the patient and patient's family.

Effective communication is an integral part of leadership. More effective and clear instructions from the leader during the communication process will help to eliminate variations [9]. Elimination of variations will in turn lead to improvement in patient flow during the discharge process [9]. All participants expressed concerns that in most instances patients and patients' families received different instructions from members of the team. R1 felt a clear instruction to patients and patients' families from a doctor acting as a team leader would alleviate this concern and improve patients' understanding of the expected date of discharge and discharge process. Variations in working time shifts, and team members responsible for the patients care were also seen as an obstacle to the discharge process by R2. Eliminations of variables have been seen as the most effective way to create a well-organised discharge process ${ }^{9}$. R3 also mentioned how the "off-set of shift telemetry, (where) they do 12- hour shifts and most of everybody else in the department do 8-hour shifts" profoundly disrupts effective communication. This experience was shared by R1 and R2, both confirmed that this variation had affected discharge planning.

All the respondents agreed that information of the expected date of discharge is best to be given to patient and patient's family before the operative procedure and to be communicated in a team brief between members of the multidisciplinary team at day one of the patient's admission into hospital. R1 and R2 also recommended that one channel of communication prior to the surgery preferably from the leader of the team will be most effective. The hospital has developed schedule to estimate the total time required for a patient for the operation.

Leadership is described as a link between the individual and the organisation. From this concept, effective leadership will evaluate behaviour within the organisation and set ways to provide effective collaboration between management and employee to lead and manage the implementation of changes throughout the organisation.

When a participant asked, "If there are different leadership strategies that work better in a given situation", R1 \& R2 both gave specific examples about a team leader working at their institution who uses "flexible approach" in leading the team. R1 specifically said 'a physiotherapist team leader at her department is known to know all the policies and protocol and generally consulted by other physiotherapists 
and get utilised to perform different roles when there is a need'. R1 believed this has led to a better discharge process. R2 brought a similar example and added 'when there is a concern about poor performing member of the team, team leader always offers help and advice to explore the reasons of poor performance and assess any training needs'.

When asked 'how current leadership strategies reduce discharge inefficiencies 'All participants suggested doing frequent ward rounds. R3 explained how frequent rounds help their team to explore patients' needs and circumstances more efficiently and said 'sometimes we realise home oxygen therapy is needed by the patients at day two or three of their admission. Some patients only give information when we reassessed them throughout their in-patient stay'. R1 recommended that it is important to have an adaptable and flexible approach particularly when deciding on the frequency of round as long this does not affect the delivery of efficient service throughout the ward'. R2 reported that, 'rigorous rounds and reviews allows clear understanding of patient's needs and hence better discharge planning. R2 also reported on their successful experience in early discharge process following THA through communication with patients and patient's family by a team leader or a surgeon who operate on the patients about estimated discharge date from the first moment of patient's arrival to the hospital. Holland et al. (2016) [10] supported exploring patients' needs on admission to facilitate the discharge process. Shepperd et al. (2013) [11] also stressed the importance of focusing of estimated LOS at first patients contacted by the team leader. Although healthcare organisations adapt a customised discharge plan for each patient through information leaflets and discharge documentation, these will not guarantee that patients or patients' caregiver will understand them in a clear and understandable manner. Joynt et al. (2014) [12] suggested that effective leadership will provide skills and expertise to coordinate these discharge instructions and will ensure that the process will be explained through an admitting team Therefore, documentation and information leaflets should not be seen as a replacement to direct patients-leader's communication.

The results of the statistical analysis of the data collected is presented in Table 2. The causes of the readmission complications were due to wound bleeding, Oedema, dislocation and revision of THR required. Data analysis shows improvements after intervention of the doctors of the hospital.

\section{Discussion}

The following findings of the interviews showed effective leadership and communications are the key factors that influence the LOS of the patients in the hospital and these are in line with the literature [12]. Effective leadership streamlines the discharge process through effective communication skills and provision of clear protocol and guidance from patients' admission to the discharge. The need for effective leadership also correlated with the literature. There is a need for flexibility and better organisation to provide an effective discharge planning. The findings from this research also found, implementing collaborative changes will ensure patients can leave the hospital in a timely and costeffective way.

This research found two important elements of the strategy to help reduce LOS of patient at the hospital. The two elements include effective communication and effective leadership. The effective communication has three components, which include communication between doctors team, the patients and their families, communication between doctors and team members including support staff, and communication between doctors and the nurses. Effective leadership is a combination of many leadership styles. The effective leadership approach is a flexible approach where different leadership styles are adopted according to the situation at hand. Effective communication and effective leadership reduce the LOS of the patients at the hospital considerably

The findings from this research are extremely relevant and contribute significantly to the body of literature on reducing LOS in patients following THA. Nevertheless, as with most studies, this study creates a number of pertinent questions to provide better clarity on this issue. Therefore, the authors of this research have provided some recommendations from this research. The first recommendation would be to conduct further larger, prospective studies, using a greater number of hospitals with different clinical specialities. This is particularly relevant, as it is important to include different sized hospitals, as the discharge process may be different and may change according to the size of the hospital. Generally, when institution becomes larger, there is a potential for breakdowns across many domains, particularly communication.

The results about the effects of specific style of leadership on the length of stay after THA are in support of this hypothesis i.e. effective leadership practices lead to a decrease in the LOS. The study included 3 hospitals and 114 sample patient data studied with and without intervention at a gap of 6 months duration. The length of stay, age, gender, ASA grade, readmissions and reasons for delayed discharge were considered during the study to analyse the effects of leadership.

Considerable decrease in the patients' length of stay following THA after the intervention based on the two themes i.e. effective communication and leadership was noticed. The instrument developed by the leader led to a decrease of $25 \%$ in the length of the stay. Additionally, the fraction of patients with a stay of less than or equal to 3 days was enhanced by $23 \%$ and about $26 \%$ of the patients stayed for less than 2 days, reassuring the effectiveness of the leadership technique suggested in this study.

Table 2. Analysis of patient stay in the hospital before and after the intervention

\begin{tabular}{|c|c|c|c|c|}
\hline \multirow[b]{2}{*}{ S. No. } & \multirow[b]{2}{*}{ Aspects of patient stay } & \multirow[b]{2}{*}{$\begin{array}{l}\text { Before intervention } \\
\text { period }(6-10-2016 \\
\text { to } 27-3-2017)\end{array}$} & \multirow{2}{*}{$\begin{array}{c}\text { Results } \\
\text { After intervention } \\
\text { period (27-3-2017 to 6-10-2017) }\end{array}$} & \multirow[b]{2}{*}{ Improvement } \\
\hline & & & & \\
\hline 1 & $\begin{array}{l}\text { Median length of the inpatient stay } \\
\text { (days) }\end{array}$ & 4 & 3 & $25 \%$ \\
\hline 2 & $\begin{array}{l}\text { Median length of the inpatient stay } \\
\text { (days) }(95 \% \mathrm{Cl})\end{array}$ & 4.6 & 3.8 & $17.39 \%$ \\
\hline 3 & $\begin{array}{l}\text { Percentage of patients staying } \leq \\
3 \text { days }\end{array}$ & $42.6 \%$ & $65.2 \%$ & $53.05 \%$ \\
\hline 4 & $\begin{array}{c}\text { Percentage of patients staying } \leq 2 \\
\text { days }\end{array}$ & N.A & $26.0 \%$ & \\
\hline 5 & Number of readmissions & 4 & 0 & $100 \%$ \\
\hline
\end{tabular}


Surprisingly the number of readmissions also decreased to none (by $100 \%$ ) after the intervention suggesting improvement in the quality of treatment by the instrument.

The instrument based on effective leadership strategy which included enhancing communication, saved the time and resources of the hospitals considerably by decreasing the length of stays and avoiding readmissions altogether. Since there was no additional investment in reducing the length of stay of the patient, the hypothesis stands proved.

\section{Conclusion}

In conclusion, effective communication and effective leadership are important factors to reduce the LOS of patient in hospital. In addition to these two focal points, a total holistic management approach is necessary, which includes appropriate leadership style according to the needs of the patients and the situation. Flexible leadership approach is imperative within hospital management for reducing the LOS of patients who have recently undergone a hospital admission for THA.

\section{References}

1. Epstein AM, Read J Leighton, Hoefer M (1987) The relation of body weight to length of stay and charges for hospital services for patients undergoing elective surgery: a study of two procedures. Am J Public Health 77: 993-997. [Crossref]

2. Epps CD (2004) Length of stay, discharge disposition and hospital charge predictors. AORN J 79: 975-976.
3. Ogonda L, Wilson R, Archbold P, Lawlor M, Humphreys P, et al. (2005) A minimalincision technique in total hip arthroplasty does not improve early postoperative outcomes. A prospective, randomized, controlled trial. J Bone Joint Surg Am 87: 701710. [Crossref]

4. Sibia US, Waite KA, Callanan MA, Park AE, King PJ (2017) Do shorter lengths of stay increase readmissions after total joint replacements? Arthroplasty Today 3: 51-55. [Crossref]

5. Boonstra JJ (2012) Cultural change and leadership in organizations: A practical guide to successful organizational change. Hoboken, NJ: Wiley.

6. Hurwitz JE, Lee JA, Lopiano KK, McKinley SA, Keesling J (2014) A flexible simulation platform to quantify and manage emergency department crowding. $B M C$ Med Inform Decis Mak 14: 50. [Crossref]

7. Ganong L, Coleman M (2014) Qualitative research on family relationships. J Social Personal Relationships 31: 451-459.

8. Greysen SR, Schiliro D, Horwitz LI, Curry L, Bradley EH (2012) Out of sight, out of mind: House staff perceptions of quality limiting factors in discharge care at teaching hospitals. J Hosp Med 7: 376-381. [Crossref]

9. Soong C, High S, Morgan MW, Ovens H (2013) A novel approach to improving emergency department consultant response times. BMJ Qual Saf 22: 299-305. [Crossref]

10. Howell EF (2013) The origins of psychoanalysis in a case of probable DID: A review of a dream of undying fame: how freud betrayed his mentor and invented psychoanalysis. $J$ Trauma Dissociation.

11. Shepperd S, Lannin NA, Clemson LM, McCluskey A, Cameron ID (2013) Discharge planning from hospital to home. Cochrane Database Syst Rev Art. No: CD000313. [Crossref]

12. Joynt KE, Orav EJ, \& Jha, A. K. (2014). Association between hospital conversions to for-profit status and clinical and economic outcomes. JAMA 312: 1644-1652. [Crossref]

Copyright: (C2019 Radha S. This is an open-access article distributed under the terms of the Creative Commons Attribution License, which permits unrestricted use, distribution, and reproduction in any medium, provided the original author and source are credited. 